\title{
SOME CONGRUENCES INVOLVING CATALAN, PELL AND FIBONACCI NUMBERS
}

\author{
S. KOPARAL ${ }^{1 *}$ AND N. ÖMÜR ${ }^{1}$ \\ ${ }^{1}$ Kocaeli University Mathematics Department 41380 İzmit Kocaeli Turkey. \\ *Corresponding author. E-mail: sibel.koparal@kocaeli.edu.tr
}

\section{DOI: 10.20948/mathmontis-2020-48-2}

Summary. In this paper, using some special numbers and combinatorial identities, we show some interesting congruences: for a prime $p>5$,

$$
\begin{gathered}
\sum_{k=0}^{(p-1) / 2} \frac{C_{k}}{8^{k}(k+1)} \equiv 2^{(7-p) / 2} \frac{P_{p}}{p}-4\left(\frac{2}{p}\right)+8-\frac{2^{p+2}}{p}(\bmod p), \\
\sum_{k=1}^{(p+1) / 2} \frac{C_{k-1}}{4^{k} k(k+1)} \equiv-q_{p}(2)+\frac{5}{6}(\bmod p), \\
\sum_{k=1}^{(p+1) / 2} \frac{C_{k-1}}{(-16)^{k} k(k+1)(k+2)} \equiv \frac{L_{3 p}}{2^{p+2} p}-\frac{2^{p-2}}{p}+\frac{5}{2}\left(\frac{5}{p}\right)-\frac{91}{40}(\bmod p),
\end{gathered}
$$

where $C_{n}, L_{n}$ and $P_{n}$ are the $n$th Catalan number, the $n$th Lucas number and the $n$th Pell number, respectively. $\left(\frac{.}{p}\right)$ denotes the Legendre symbol.

\section{INTRODUCTION}

The Fibonacci sequence $\left\{F_{n}\right\}$ and the Lucas sequence $\left\{L_{n}\right\}$ are defined by the following recursions:

$$
F_{n+1}=F_{n}+F_{n-1} \quad \text { and } \quad L_{n+1}=L_{n}+L_{n-1}, n>0 \text {, }
$$

where $F_{0}=0, F_{1}=1$ and $L_{0}=2, L_{l}=1$, respectively. If $\alpha$ and $\beta$ are the roots of equation $x^{2}-x-1=0$, the Binet formulas of the sequences $\left\{F_{n}\right\}$ and $\left\{L_{n}\right\}$ have the forms

$$
F_{n}=\frac{\alpha^{n}-\beta^{n}}{\alpha-\beta} \quad \text { and } \quad L_{n}=\alpha^{n}+\beta^{n} \text {, }
$$

respectively.

The Pell sequence $\left\{P_{n}\right\}$ and the Pell-Lucas sequence $\left\{Q_{n}\right\}$ are defined recursively by

$$
P_{n+1}=2 P_{n}+P_{n-1} \quad \text { and } \quad Q_{n+1}=2 Q_{n}+Q_{n-1}, n>0,
$$

in which $P_{0}=0, P_{1}=1$ and $Q_{0}=Q_{1}=2$, respectively. If $\gamma$ and $\delta$ are the roots of equation 
$x^{2}-2 x-1=0$, the Binet formulas of the sequences $\left\{P_{n}\right\}$ and $\left\{Q_{n}\right\}$ have the forms

$$
P_{n}=\frac{\gamma^{n}-\delta^{n}}{\gamma-\delta} \quad \text { and } \quad Q_{n}=\gamma^{n}+\delta^{n}
$$

respectively.

The harmonic numbers have interesting applications in many fields of mathematics, such as number theory, combinatorics, analysis and computer science. Harmonic numbers $H_{n}$ are defined as for a positive integer $n$

$$
H_{n}=\sum_{k=1}^{n} \frac{1}{k}
$$

where $H_{0}=0$. The first few harmonic numbers are $1, \frac{3}{2}, \frac{11}{6}, \frac{25}{12}, \ldots$

Some elementary combinatorial properties of the Catalan numbers are given in $[2,6,8]$. The Catalan numbers are given by

$$
C_{n}=\frac{1}{n+1}\left(\begin{array}{c}
2 n \\
n
\end{array}\right)=\left(\begin{array}{c}
2 n \\
n
\end{array}\right)-\left(\begin{array}{c}
2 n \\
n+1
\end{array}\right), n \in N
$$

For a prime $p$ and an integer $a$ with $p \nmid a$, we write the Fermat quotient $q_{p}(a)=\left(a^{p-1}-1\right) / p$. For an odd prime $p$ and an integer $a,\left(\frac{a}{p}\right)$ denotes the Legendre symbol defined by

$$
\left(\frac{a}{p}\right)=\left\{\begin{array}{cc}
0 & \text { if } p \mid a, \\
1 & \text { if } a \text { is a quadratic residue } \bmod p, \\
-1 & \text { if } a \text { is a quadratic nonresidue } \bmod p .
\end{array}\right.
$$

In [3], E. Lehmer showed that for prime $p>3$,

In [9], Z.W. Sun obtained that

$$
H_{(p-1) / 2} \equiv-2 q_{p}(2)(\bmod p) .
$$

$$
\sum_{k=0}^{(p-1) / 2} \frac{1}{m^{k}}\left(\begin{array}{c}
2 k \\
k
\end{array}\right) \equiv\left(\frac{m(m-4)}{p}\right)(\bmod p)
$$

and

$$
\sum_{k=0}^{(p-1) / 2} \frac{1}{m^{k}(k+1)}\left(\begin{array}{c}
2 k \\
k
\end{array}\right) \equiv \frac{m}{2}-\frac{m-4}{2}\left(\frac{m(m-4)}{p}\right)(\bmod p),
$$

where $p$ is an odd prime and $m$ is any integer not divisible by $p$.

Let $p$ be a fixed prime $>3$. Define

$$
q(x)=\frac{x^{p}-(x-1)^{p}-1}{p} \quad \text { and } \quad G_{n}(x)=\sum_{k=1}^{p-1} \frac{x^{k}}{k^{n}},
$$

where $x$ is variable.

In [1], A. Granville showed that 


$$
\begin{gathered}
q(x) \equiv-G_{1}(x)(\bmod p), \\
G_{2}(x) \equiv G_{2}(1-x)+x^{p} G_{2}(1-1 / x)(\bmod p), \\
(q(x))^{2} \equiv-2 x^{p} G_{2}(x)-2\left(1-x^{p}\right) G_{2}(1-x)(\bmod p) .
\end{gathered}
$$

It was proved in [1] (the last expression on page 3) that for any integer $n>1$,

$$
\sum_{k=1}^{n-1}\left(\begin{array}{l}
n-1 \\
k-1
\end{array}\right) \frac{(-x)^{k}}{k^{2}}=\frac{1}{n} \sum_{k=1}^{n-1} \frac{(1-x)^{k}-1}{k}+\frac{(1-x)^{n}-(-x)^{n}-1}{n^{2}} .
$$

In [7], Z.H. Sun obtained the following congruences: for an odd prime $p$ and $G_{n}(x) \in Z_{p}[x]$,

$$
G_{2}(x) \equiv \frac{1}{p}\left(\frac{1+(x-1)^{p}-x^{p}}{p}-\sum_{k=1}^{p-1} \frac{(1-x)^{k}-1}{k}\right)+p \sum_{r=2}^{p-1} \frac{x^{r}}{r^{2}} \sum_{s=1}^{r-1} \frac{1}{s}\left(\bmod p^{2}\right),
$$

and for a prime $p>3$ and $n \in N$,

$$
n p G_{n+1}(x) \equiv(-1)^{n} x^{p} G_{n}(1 / x)-G_{n}(x)\left(\bmod p^{2}\right) .
$$

In [10], Z.W. Sun showed that for a prime $p>3$,

$$
\sum_{k=1}^{p-1} \frac{H_{k} L_{k}}{k} \equiv 0(\bmod p) \quad \text { and } \quad \sum_{k=1}^{p-1} \frac{H_{k} F_{k}}{k} \equiv \frac{2}{p} \sum_{k=1}^{p-1} \frac{F_{k}}{k}(\bmod p) .
$$

In [5], H. Pan and Z.W. Sun obtained that for a prime $p>5$,

$$
\sum_{k=1}^{p-1} \frac{L_{k}}{k^{2}} \equiv O(\bmod p)
$$

and for a prime $p \neq 2,5$,

$$
\sum_{k=0}^{p^{a}-1}(-1)^{k}\left(\begin{array}{c}
2 k \\
k
\end{array}\right) \equiv\left(\frac{p^{a}}{5}\right)\left(\begin{array}{c}
1-2 F_{p^{a}-\left(\frac{p^{a}}{5}\right)}
\end{array}\right)\left(\bmod p^{3}\right),
$$

where $a$ is a positive integer.

In [4], S. Mattarei and R. Tauraso showed that for a prime $p>3$,

$$
\sum_{k=1}^{p-1}\left(\begin{array}{c}
2 k \\
k
\end{array}\right) \frac{x^{k}}{k^{2}} \equiv 2\left(G_{2}(\lambda)+G_{2}(\mu)\right)(\bmod p),
$$

where $\lambda=\frac{1}{2}(1+\sqrt{1-4 x})$ and $\mu=\frac{1}{2}(1-\sqrt{1-4 x})$.

\section{SOME CONGRUENCES INVOLVING SPECIAL NUMBERS}

In this section, we will give the congruences involving some special numbers. Now, we give the following lemma for further use.

Lemma 1. Let $n$ be any positive integer. Then

$$
\sum_{k=1}^{n}\left(\begin{array}{c}
n-1 \\
k-1
\end{array}\right) \frac{(-x)^{k+1}}{k^{2}(k+1)}=\frac{1}{n} \sum_{k=1}^{n-1} \frac{(1-x)^{k+1}-1}{k(k+1)}+\frac{(1-x)^{n+1}-1}{n^{2}(n+1)}+\frac{x}{n} H_{n}
$$


and

$$
\sum_{k=1}^{n}\left(\begin{array}{c}
n-1 \\
k-1
\end{array}\right) \frac{(-x)^{k+2}}{k^{2}(k+1)(k+2)}=\frac{1}{n} \sum_{k=1}^{n-1} \frac{(1-x)^{k+2}-1}{k(k+1)(k+2)}+\frac{(1-x)^{n+2}-1}{n^{2}(n+1)(n+2)}+\frac{x}{n+1}-\frac{x^{2}}{2 n} H_{n}
$$

Proof. From Binomial Theorem, we have

$$
\begin{aligned}
& \sum_{k=1}^{n-1} \frac{(1-x)^{k+1}-1}{k(k+1)}+\frac{(1-x)^{n+1}-1}{n(n+1)}+x H_{n} \\
& =\sum_{k=1}^{n} \frac{(1-x)^{k+1}-1}{k(k+1)}+x \sum_{k=1}^{n} \frac{1}{k}=\sum_{k=1}^{n} \frac{(1-x)^{k+1}-1+(k+1) x}{k(k+1)} \\
& =\sum_{k=2}^{n+1} \frac{(1-x)^{k}-1+k x}{k(k-1)}=\sum_{k=2}^{n+1} \frac{1}{k(k-1)} \sum_{j=2}^{k}\left(\begin{array}{c}
k \\
j
\end{array}\right)(-x)^{j}=\sum_{k=2}^{n+1} \frac{1}{k-1} \sum_{j=2}^{k} \frac{(-x)^{j}}{j}\left(\begin{array}{c}
k-1 \\
j-1
\end{array}\right) \\
& =\sum_{k=2}^{n+1} \sum_{j=2}^{k} \frac{(-x)^{j}}{j(j-1)}\left(\begin{array}{c}
k-2 \\
j-2
\end{array}\right)=\sum_{j=2}^{n+1} \frac{(-x)^{j}}{j(j-1)} \sum_{\mathrm{k}=\mathrm{j}}^{\mathrm{n}+1}\left(\begin{array}{c}
k-2 \\
j-2
\end{array}\right) .
\end{aligned}
$$

From the equality $\sum_{k=j}^{n+1}\left(\begin{array}{c}k-2 \\ j-2\end{array}\right)=\left(\begin{array}{c}n \\ j-1\end{array}\right)$, we get

$$
\begin{aligned}
& \sum_{k=1}^{n-1} \frac{(1-x)^{k+1}-1}{k(k+1)}+\frac{(1-x)^{n+1}-1}{n(n+1)}+x H_{n} \\
& =\sum_{j=2}^{n+1}\left(\begin{array}{c}
n \\
j-1
\end{array}\right) \frac{(-x)^{j}}{j(j-1)}=\sum_{j=1}^{n}\left(\begin{array}{c}
n \\
j
\end{array}\right) \frac{(-x)^{j+1}}{j(j+1)}=n \sum_{j=1}^{n}\left(\begin{array}{c}
n-1 \\
j-1
\end{array}\right) \frac{(-x)^{j+1}}{j^{2}(j+1)} .
\end{aligned}
$$

Thus, the first identity is obtained. Similarly, we obtain other identity.

For a prime $p$ and a rational number $x=\frac{a}{b}$ written in reduced form, $x \equiv 0(\bmod p)$ if and only if $a$ is divisible by $p$.

Theorem 1. Let $p$ be an odd prime. For any rational number $x$ such that $x^{-1} \not \equiv O(\bmod p)$,

$$
\sum_{k=0}^{(p-1) / 2} \frac{C_{k}}{k+1} x^{k+1} \equiv 1-(\lambda-\mu)^{p+1}+\frac{2^{p-1}}{p}\left(\lambda^{p}+\mu^{p}-1\right)(\bmod p),
$$

where $\lambda=\frac{1}{2}(1+\sqrt{1-4 x})$ and $\mu=\frac{1}{2}(1-\sqrt{1-4 x})$.

Proof. Replacing $n$ and $x$ by $(p+1) / 2$ and $4 x$ in (1.2), respectively, we have 


$$
\frac{p+1}{2} \sum_{k=0}^{(p-1) / 2}\left(\begin{array}{c}
(p-1) / 2 \\
k
\end{array}\right) \frac{(-4 x)^{k+1}}{(k+1)^{2}}=\sum_{k=1}^{(p-1) / 2} \frac{(1-4 x)^{k}}{k}+2 \frac{(1-4 x)^{(p+1) / 2}-1}{p+1}-H_{(p-1) / 2} .
$$

From the congruences $\left(\begin{array}{c}(p-1) / 2 \\ k\end{array}\right)(-4)^{k} \equiv\left(\begin{array}{c}2 k \\ k\end{array}\right)(\bmod p)$ for $k=1, \ldots,(p-1) / 2$,

$\frac{1}{p+k} \equiv \frac{1}{k}(\bmod p)$ for $k=1, \ldots, p-1$ and $(1.1)$, we write

$$
-2 \sum_{k=0}^{(p-1) / 2}\left(\begin{array}{c}
2 k \\
k
\end{array}\right) \frac{x^{k+1}}{(k+1)^{2}} \equiv 2\left((1-4 x)^{(p+1) / 2}-1\right)+\sum_{k=1}^{(p-1) / 2} \frac{(1-4 x)^{k}}{k}+2 q_{p}(2)(\bmod p) .
$$

By congruence $\left(\begin{array}{c}p-1 \\ k\end{array}\right) \equiv(-1)^{k}(\bmod p)$ for $k=1, \ldots, p-1$, we get

$$
\begin{aligned}
\sum_{k=1}^{(p-1) / 2} \frac{(1-4 x)^{k}}{k} & \equiv-\sum_{k=1}^{(p-1) / 2}\left(\begin{array}{c}
p-1 \\
2 k-1
\end{array}\right) \frac{(1-4 x)^{k}}{k} \\
& =-\frac{2}{p} \sum_{k=1}^{(p-1) / 2}\left(\begin{array}{c}
p \\
2 k
\end{array}\right)(1-4 x)^{k}=\frac{2-(1+\sqrt{1-4 x})^{p}-(1-\sqrt{1-4 x})^{p}}{p}(\bmod p) .
\end{aligned}
$$

Substituting (2.3) into (2.2), we have

$$
\begin{aligned}
\sum_{k=0}^{(p-1) / 2} \frac{C_{k}}{k+1} x^{k+1} & =\sum_{k=0}^{(p-1) / 2}\left(\begin{array}{c}
2 k \\
k
\end{array}\right) \frac{x^{k+1}}{(k+1)^{2}} \\
& \equiv 1-(1-4 x)^{(p+1) / 2}-q_{p}(2)-\frac{(\sqrt{1-4 x}-1)^{p}-(\sqrt{1-4 x}+1)^{p}+2}{2 p} \\
& =1-(\lambda-\mu)^{p+1}+\frac{2^{p-1}}{p}\left(\lambda^{p}+\mu^{p}-1\right)(\bmod p) .
\end{aligned}
$$

Thus, this concludes the proof.

From Theorem 1, we immediately deduce the following results.

Corollary 1. Let $p$ be an odd prime. Then

$$
\begin{gathered}
\sum_{k=0}^{(p-1) / 2} \frac{C_{k}}{4^{k}(k+1)} \equiv 4\left(1-q_{p}(2)\right) \quad(\bmod p), \\
\sum_{k=0}^{(p-1) / 2} \frac{(-1)^{k} C_{k}}{k+1} \equiv 2^{p-1} \frac{1-L_{p}}{p}+5\left(\frac{5}{p}\right)-1 \quad(\bmod p), \\
\sum_{k=0}^{(p-1) / 2} \frac{C_{k}}{8^{k}(k+1)} \equiv 2^{(7-p) / 2} \frac{P_{p}}{p}-4\left(\frac{2}{p}\right)+8-\frac{2^{p+2}}{p}(\bmod p),
\end{gathered}
$$




$$
\begin{gathered}
\sum_{k=0}^{(p-1) / 2} \frac{C_{k}}{(-4)^{k}(k+1)} \equiv-\frac{2 Q_{p}}{p}+\frac{2^{p+1}}{p}+8\left(\frac{2}{p}\right)-4(\bmod p), \\
\sum_{k=0}^{(p-1) / 2} \frac{C_{k}}{(-16)^{k}(k+1)} \equiv-\frac{2^{3-p}}{p} L_{3 p}+\frac{2^{p+3}}{p}+20\left(\frac{5}{p}\right)-16 \quad(\bmod p),
\end{gathered}
$$

and for $p>3$

$$
\sum_{k=0}^{(p-1) / 2} \frac{C_{k}}{9^{k}(k+1)} \equiv \frac{2^{p-1}}{p}\left(\frac{L_{2 p}}{3^{p-2}}-9\right)-5\left(\frac{5}{p}\right)+9 \quad(\bmod p) .
$$

Proof. For the proof (2.4), considering $x=-1$ in Theorem 1, we have

$$
\begin{aligned}
& \sum_{k=0}^{(p-1) / 2} \frac{C_{k}}{(-1)^{k+1}(k+1)} \\
\equiv & 1-5^{(p+1) / 2}+\frac{2^{p-1}}{p}\left(\left(\frac{1+\sqrt{5}}{2}\right)^{p}+\left(\frac{1-\sqrt{5}}{2}\right)^{p}-1\right) \equiv 1-5\left(\frac{5}{p}\right)+\frac{2^{p-1}}{p}\left(L_{p}-1\right)(\bmod p) .
\end{aligned}
$$

Similarly, we obtain other five congruences of Corollary 1.

Theorem 2. Let $p>3$ be a prime. For any rational number $x$ such that $x^{-1} \not \equiv O(\bmod p)$,

$$
\sum_{k=1}^{(p+1) / 2} \frac{C_{k-1}}{k(k+1)} x^{k+1} \equiv \frac{2^{p-1} x}{p}\left(\lambda^{p}+\mu^{p}-1\right)-\frac{8 x+1}{12}(\lambda-\mu)^{p+1}+\frac{6 x+1}{12}(\bmod p),
$$

where $\lambda$ and $\mu$ are defined as before.

Proof. By the first identity of Lemma 1, we have

$$
\sum_{k=0}^{n-1}\left(\begin{array}{c}
n-1 \\
k
\end{array}\right) \frac{(-x)^{k+2}}{(k+1)^{2}(k+2)}=\frac{1}{n} \sum_{k=1}^{n-1} \frac{(1-x)^{k+1}-1}{k(k+1)}+\frac{(1-x)^{n+1}-1}{n^{2}(n+1)}+\frac{x}{n} H_{n} .
$$

Replacing $n$ and $x$ by $(p+1) / 2$ and $4 x$ in the aboving sum, respectively, we have $\sum_{k=0}^{(p-1) / 2}\left(\begin{array}{c}(p-1) / 2 \\ k\end{array}\right) \frac{(-4 x)^{k+2}}{(k+1)^{2}(k+2)}=\frac{2}{p+1} \sum_{k=1}^{(p-1) / 2} \frac{(1-4 x)^{k+1}-1}{k(k+1)}+8 \frac{(1-4 x)^{(p+3) / 2}-1}{(p+1)^{2}(p+3)}+\frac{8 x}{p+1} H_{(p+1) / 2}$.

From the congruences $\left(\begin{array}{c}(p-1) / 2 \\ k\end{array}\right)(-4)^{k} \equiv\left(\begin{array}{c}2 k \\ k\end{array}\right)(\bmod p)$ for $k=1, \ldots,(p-1) / 2$, $\frac{1}{p+k} \equiv \frac{1}{k}(\bmod p)$ for $k=1, \ldots, p-1$ and $(1.1)$, we write 


$$
\begin{aligned}
& \sum_{k=0}^{(p-1) / 2}\left(\begin{array}{c}
2 k \\
k
\end{array}\right) \frac{x^{k+2}}{(k+1)^{2}(k+2)} \\
& \equiv \frac{(1-4 x)-1}{8} \sum_{k=1}^{(p-1) / 2} \frac{(1-4 x)^{k}}{k}+\frac{1}{4(p+1)}-\frac{(1-4 x)^{(p+1) / 2}}{4(p+1)}+\frac{(1-4 x)^{(p+3) / 2}}{6}+x-\frac{x}{2}-x q_{p}(2)-\frac{1}{6}(\bmod p) .
\end{aligned}
$$

From the congruences $(2.3)$ and $\frac{1}{p+1} \equiv 1(\bmod p)$, we have

$$
\begin{aligned}
& \sum_{k=1}^{(p+1) / 2} \frac{C_{k-1}}{k(k+1)} x^{k+1} \\
\equiv & -\frac{x}{2 p}\left(2-(1+\sqrt{1-4 x})^{p}-(1-\sqrt{1-4 x})^{p}\right)-\frac{(1-4 x)^{(p+1) / 2}}{4}+\frac{(1-4 x)^{(p+3) / 2}}{6}+\frac{x}{2}-x q_{p}(2)-\frac{5}{2} \\
= & -\frac{x}{2 p}\left(2^{p}-(1+\sqrt{1-4 x})^{p}-(1-\sqrt{1-4 x})^{p}\right)-\frac{(1-4 x)^{(p+1) / 2}}{4}+\frac{(1-4 x)^{(p+3) / 2}}{6}+\frac{x}{2}-\frac{5}{2} \quad(\bmod p) .
\end{aligned}
$$

Thus, the desired result is obtained.

From the above equation, we get the assertion of the theorem.

As an immediate consequence of Theorem 2, we obtain the following result.

Corollary 2. Let $p>3$ be a prime. Then

$$
\begin{gathered}
\sum_{k=1}^{(p+1) / 2} \frac{C_{k-1}}{4^{k} k(k+1)} \equiv-q_{p}(2)+\frac{5}{6} \quad(\bmod p), \\
\sum_{k=1}^{(p+1) / 2} \frac{(-1)^{k} C_{k-1}}{k(k+1)} \equiv 2^{p-1} \frac{L_{p}-1}{p}-\frac{35}{12}\left(\frac{5}{p}\right)+\frac{5}{12} \quad(\bmod p), \\
\sum_{k=1}^{(p+1) / 2} \frac{C_{k-1}}{8^{k} k(k+1)} \equiv \frac{P_{p}}{2^{(p-1) / 2} p}-\frac{2^{p-1}}{p}-\frac{4}{3}\left(\frac{1}{2}\right)^{(p+1) / 2}+\frac{7}{6} \quad(\bmod p), \\
\sum_{k=1}^{(p+1) / 2} \frac{C_{k-1}}{9^{k} k(k+1)} \equiv \frac{2^{p-1}}{p}\left(\frac{L_{2 p}}{\left.3^{p}-1\right)}-\frac{85}{108}\left(\frac{5}{p}\right)+\frac{5}{4}(\bmod p),\right. \\
\sum_{k=1}^{(p+1) / 2} \frac{C_{k-1}}{(-4)^{k} k(k+1)} \equiv \frac{Q_{p}-2^{p}}{2 p}-\frac{2}{3}\left(\frac{2}{p}\right)+\frac{1}{6} \quad(\bmod p),
\end{gathered}
$$

and

$$
\sum_{k=1}^{(p+1) / 2} \frac{C_{k-1}}{(-16)^{k} k(k+1)} \equiv \frac{L_{3 p}}{2^{p+1} p}-\frac{2^{p-1}}{p}+\frac{5}{6}\left(\frac{5}{p}\right)-\frac{5}{6}(\bmod p) .
$$

Theorem 3. Let $p>5$ be a prime. For any rational number $x$ such that $x^{-1} \not \equiv O(\bmod p)$, 


$$
\begin{aligned}
\sum_{k=1}^{(p+1) / 2} \frac{C_{k-1}}{k(k+1)(k+2)} x^{k+2} \equiv & \frac{2^{p-2}}{p} x^{2}\left(\lambda^{p}+\mu^{p}-1\right)-\frac{1}{60}(\lambda-\mu)^{p+5} \\
& +\frac{5}{96}(\lambda-\mu)^{p+3}-\frac{1}{32}(\lambda-\mu)^{p+1}+\frac{1}{8} x^{2}+\frac{1}{12} x-\frac{1}{240}(\bmod p),
\end{aligned}
$$

where $\lambda$ and $\mu$ are defined as before.

As an direct consequence of Theorem 3, we obtain the following result.

Corollary 3. Let $p>5$ be a prime. Then

$$
\begin{aligned}
& \sum_{k=1}^{(p+1) / 2} \frac{C_{k-1}}{4^{k} k(k+1)(k+2)} \equiv-\frac{1}{2} q_{p}(2)+\frac{47}{120} \quad(\bmod p), \\
& \sum_{k=1}^{(p+1) / 2} \frac{(-1)^{k} C_{k-1}}{k(k+1)(k+2)} \equiv 2^{p-2} \frac{L_{p}-1}{p}-\frac{15}{16}\left(\frac{5}{p}\right)+\frac{3}{80} \quad(\bmod p), \\
& \sum_{k=1}^{(p+1) / 2} \frac{C_{k-1}}{8^{k} k(k+1)(k+2)} \equiv \frac{P_{p}}{2^{(p+1) / 2} p}-\frac{2^{p-2}}{p}-\frac{3}{5}\left(\frac{1}{2}\right)^{(p+1) / 2}+\frac{21}{40} \quad(\bmod p), \\
& \sum_{k=1}^{(p+1) / 2} \frac{C_{k-1}}{9^{k} k(k+1)(k+2)} \equiv \frac{2^{p-2}}{p}\left(\frac{L_{2 p}}{3^{p}}-1\right)-\frac{29}{48}\left(\frac{5}{9}\right)^{(p+1) / 2}+\frac{43}{80}(\bmod p), \\
& \sum_{k=1}^{(p+1) / 2} \frac{C_{k-1}}{(-4)^{k} k(k+1)(k+2)} \equiv \frac{Q_{p}-2^{p}}{4 p}+\frac{1}{5}\left(\frac{2}{p}\right)-\frac{11}{40}(\bmod p),
\end{aligned}
$$

and

$$
\sum_{k=1}^{(p+1) / 2} \frac{C_{k-1}}{(-16)^{k} k(k+1)(k+2)} \equiv \frac{L_{3 p}}{2^{p+2} p}-\frac{2^{p-2}}{p}+\frac{5}{2}\left(\frac{5}{p}\right)-\frac{91}{40}(\bmod p) .
$$

\section{CONCLUSION}

Using combinatorial identities, some interesting congruences are investigated on the sums which include Catalan numbers. It is conceivable to extend our results using the useful technics or methods. A first question on the extension of these congruences can be viewed as generalizations on using the $q$-Binomial coefficients instead of the binomial coefficients. A second question on such extensions of these congruences can be observed that for an odd prime $p$ and any rational number $x$ such that $x^{-1} \not \equiv O(\bmod p)$, the congruences of the sums with decreasing factorials including Catalan numbers $\sum_{k=1}^{(p-1) / 2} \frac{C_{k-1}}{k^{\underline{m}}} x^{k}(\bmod p)$.

\section{REFERENCES}

[1] A. Granville, "The square of the Fermat quotient", Integers: Electron. J. Combin. Number 
Theory, 4, A22, (2004).

[2] P. Hilton and J. Pedersen, "Catalan numbers, their generalization, and their uses", The Math. Intelligencer, 13, 64-75 (1991).

[3] E. Lehmer, "On congruences involving Bernoulli numbers and the quotients of Fermat and Wilson”, Ann. of Math., 39, 350-360 (1938).

[4] S. Mattarei and R. Tauraso, "Congruences for central binomial sums and finite polylogarithms", J. Number Theory, 133, 131-157 (2013).

[5] H. Pan and Z.W. Sun, "Proof of three conjectures on congruences", Sci. China Math., 57(10), 2091-2102 (2014).

[6] L.W. Shapiro, "A Catalan triangle”, Discrete Math., 14, 83-90 (1976).

[7] Z.H. Sun, "Congruences involving Bernoulli and Euler numbers", J. Number Theory, 128, 280$312(2008)$.

[8] Z.W. Sun, "Binomial coefficients, Catalan numbers and Lucas quotients", Sci. China Math., 53(9), 2473-2488 (2010).

[9] Z.W. Sun, "Congruences involving generalized central trinomial coefficients", Sci. China Math., 57 (7), 1375-1400 (2014).

[10] Z.W. Sun, "On harmonic numbers and Lucas sequences", Publ. Math. Debrecen, 80(1-2), 25-41 (2012).

Received April 11, 2020 